\title{
Analysis of Personalized E-Learning System on the Basis of Behavioral Data Mining
}

\author{
Duoduo Liü, a, Lihua Zhang ${ }^{2, b}$ \\ ${ }^{1}$ Beijing Institute of Technology, Beijing, China \\ ${ }^{2}$ Beijing Institute of Technology, Beijing, China \\ a1297518210@qq.com, bbitzhlh@bit.edu.cn
}

Keywords: E-learning; Personalized recommendation system; Behavioral data; Interest feature

\begin{abstract}
As the network has become an important platform for people to exchange information, online learning is becoming one of the human's main channels for learning. In order to improve e-learning efficiency, the establishment of personalized recommendation system has becoming an urgent problem. At the same time, learner's interest features are the basis of personalized recommendation system. The paper introduces the data mining technology which helps to analysis the behavioral data reflect the interest features. Then it puts forward the model for personalized recommendation system on the basis of interest feature.
\end{abstract}

\section{Introduction}

Network brings opportunities to education that everyone can have the condition to acquire knowledge online. But, it's a double-edged sword for learners. Massive learning resources could mislead the learners with cognitive load. Introducing the recommendation service into education field is an effective measure to solve the problem, just like the personalized recommendation ideas in business. Notably, learners' requirements should never be neglected when make recommendation for learning resources, for that the requirements are the precondition for knowledge construction. This paper establishes a recommendation system on the basis of behavioral data mining.

\section{Data mining for e-learning behavior}

\subsection{Data mining}

E-Learning data mining is a process which will convert the data from variety of online teaching and learning system into useful information. This information can be used by teachers, students, parents and designers of E-Learning software system to achieve teaching optimization and improve the quality of learning. ${ }^{[1]}$ As a matter of fact, learning behavior data mining are mainly concentrated in the process of learning. Educational data mining has its specific value for different people.

For learners, educational data mining could help to make recommendation which include learning activities, resources, tasks, as well as the experience for learners to improve their study quality is the most useful value. Through the analysis of learner's e-learning behavior data and make assessment for different learners, the recommendation will be very reasonable. On the other hand, Educational data mining provide amount of feedback information objectively, that will make great use to educators to adjust and optimize educational decision making, improve education process, as well as the curriculum development.

There are five methods in educational data mining, including Statistical Analysis and Visualization, Cluster Analysis, Prediction, Relationship Mining, Text Mining ${ }^{[2]}$. The e-learning behavior analysis mainly based on the data recorded on e-learning system for study process, combined with the population characteristics of teachers and students. 


\subsection{E-learning behavior}

Actually, learning behavior refers to all the behavior occurred in learning environment. As the agent, learners spend time learning the digital learning resources by means of network operation in the networked learning environment, so that they can achieve their goals. Great changes have occurred compared with the traditional learning method in learning environment. E-learning environment has a profound influence on learners' psychological characteristics. ${ }^{[3]}$ Learners have full autonomy to choose their learning content in e-learning environment with their own interests.

Make analysis for e-learning behavior can help educators have a clear understanding to the interest features, in order that instructional designers can make recommendation to different learners. Currently, most researchers make e-learning behavior analysis by weblog from network teaching platform, including the web server logs and background database mining, learner access content, accessing frequency, depth and length of visit. Data mining process is often carried out by means of association rules in order to build a better organized course page and recommend page. ${ }^{[4]}$ In page set up, personal recommend will be more efficient, so a shorten distance content distribution is needed.

\subsection{Data mining procedure of e-learning behavior}

The data mining of e-learning behavior is concentrated on learning process mining. This mining can be divided in three part, including workflow, tools and algorithm flow, data flow. In the key link of data mining work flow, some tools and algorithms are needed, such as sequence analysis of SSAS, Cluster Analysis, Association Rules, UCINET. ${ }^{[5]}$

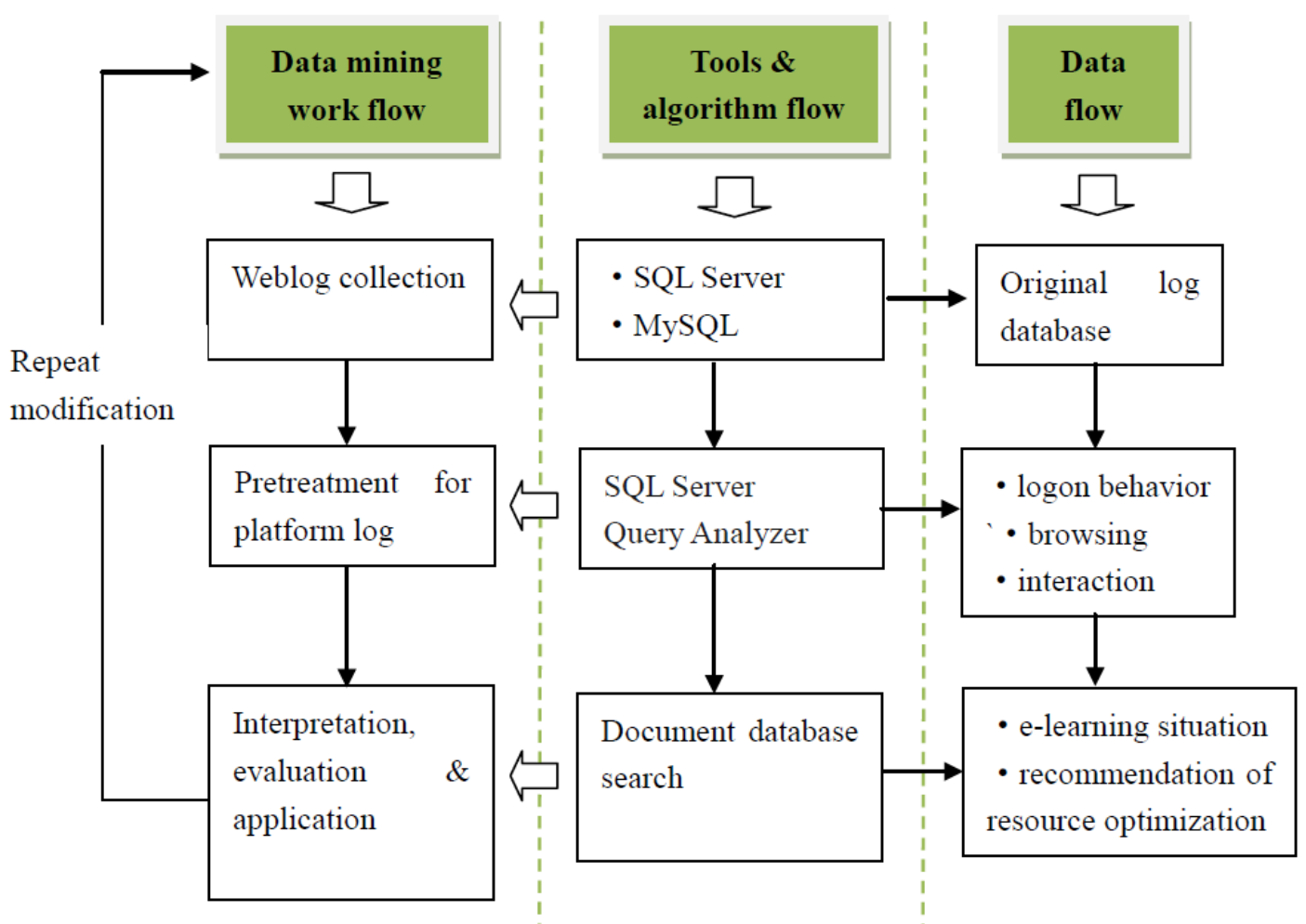

Fig. 1 Data mining model for e-learning behavior

Data collection and preprocessing, mining, models interpretation and evaluation are the core link of Data mining work flow. This part has two main tasks need to be pulled off, including collect the log from e-learning platform and behavioral analysis of learners. This is the basic sector in the whole model, which plays a decisive role for the further analysis.

Tools used by data mining part including SQL Server, SSAS, UCIMET, Excel. As algorithms and functions, Subtotals, Sequence analysis, Cluster analysis, Association Rules and network mapping are used in this part also. Specially, Sequence analysis and Cluster analysis, are mainly used for making collection and collation for similar learning behavior. Association Rules are used for mining 
related content, so that they can provide convenient recommendation by shorten the distance of similar content' distribution. ${ }^{[6]}$

When tools and algorithms are used in data mining, massive data will be formed, and that's the reason for data flow.

\section{Personalized recommendation system in e-learning}

The personalized recommendation system must be established on the basis on abundant excellent learning resources, and effectively organization and management are necessary. Only above conditions are meted, learners can enjoy the efficient resource recommendation service. Make recommendation for learners with personalized resources can improve the quality and efficiency for e-learning. In this part, the interest model will be established, on this basis, make an analysis for personalized recommendation system for e-learning.

\subsection{The interest feature model of learners}

It is necessary to take learners' personality into account when make recommendation for e-learning resources. That's to say, the e-learning resources must be meet different learners of different needs. The personalized recommendation technology derives from the development of information network, and we all know that learners have get different ways to get learning resources with intelligent terminal. Education designers should put their eyes on the establishment of learners' interest models. There are two kinds of recommendation form, including explicitly recommendation and implicit recommendation. Make resource recommendation on the basis of behavioral data mining technology, needs we have a correct command with the interest features ${ }^{[7]}$.

The establishment of interest feature can be divided into two different classes. To new registered users, they need to fill in their basic information and personal preference by their own, and all these information will be stored in a database of model. System will initialize users' interest model according to their information. Therefore, build a user interest model as shown in figure2.

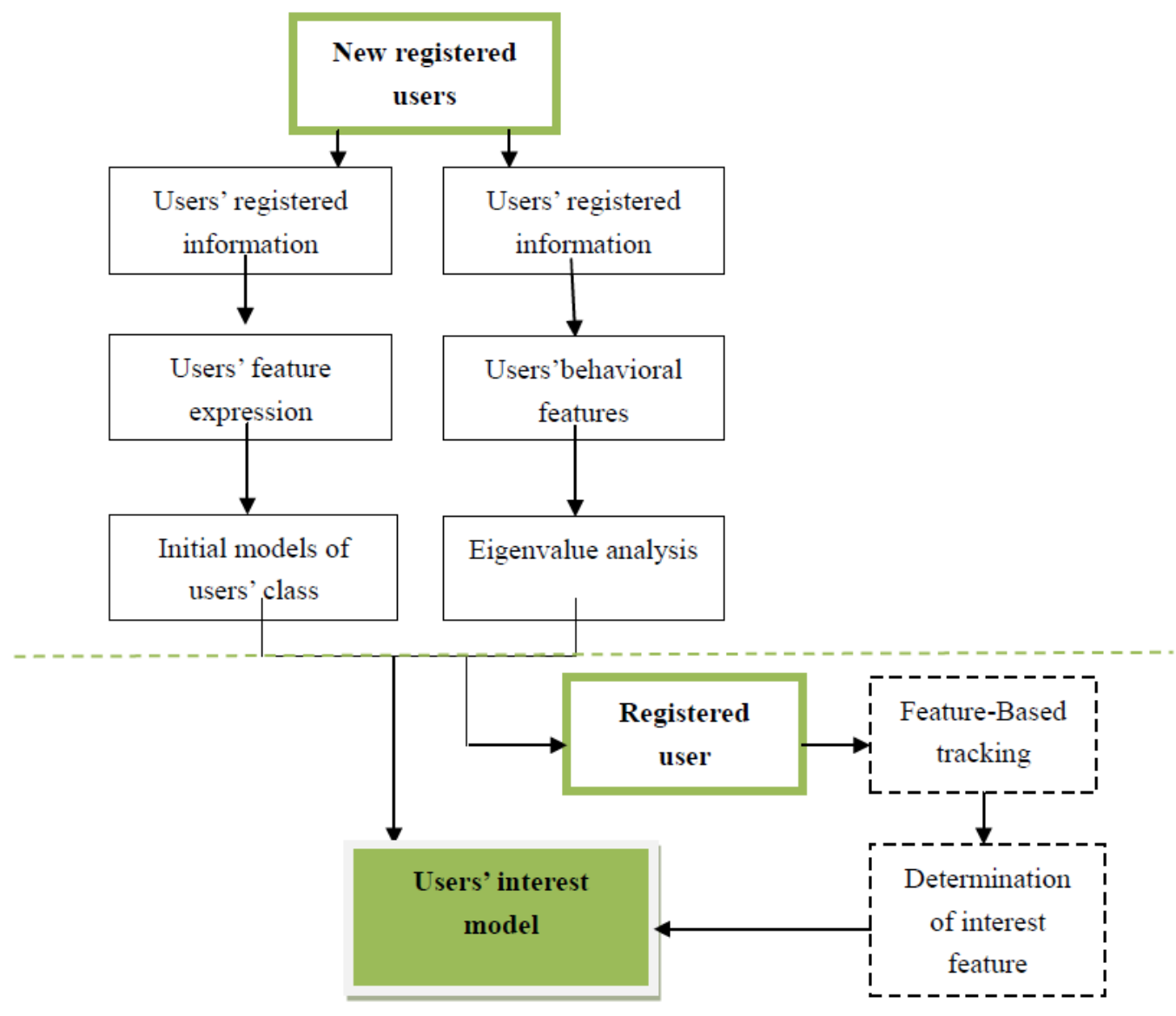

Fig. 2 Users’ interest model 
For registered learners, e-learning behavioral data mining method was taken to make analysis of learners' interest characteristics. Getting learners' characteristic words of interest by their download and other learning behavior, and this will be used as the resource of learners' interest features to update the users' interest model.

Learners' interestingness to particular subject are relatively stable in learning resources system, as well as, learner will put their passion on other subject when it is necessary for them. So, on the basis of initial interest model, the system needs to update users' interest database according to their new learning behavior, so that the model can reflect the users' accurate interest feature.

\subsection{Process of personalized learning system}

The functions of personalized learning system include these five parts: browsing resource, retrieve the resource, download, resource collection and evaluation of the resources. ${ }^{[8]}$ When the function of system was used, it will produce large amounts of data. These data will reflect the interest feature of learners accurately, and if the learners make collection and evaluation for learning resource more comprehensive, their interest feature will be reflected more accurately by the model, so the system will make a more suitable recommendation of resource.

Making learning resources recommendation under certain rules must take e-learners' learning interest feature into account. Build a personalized recommendation system as figure 3:

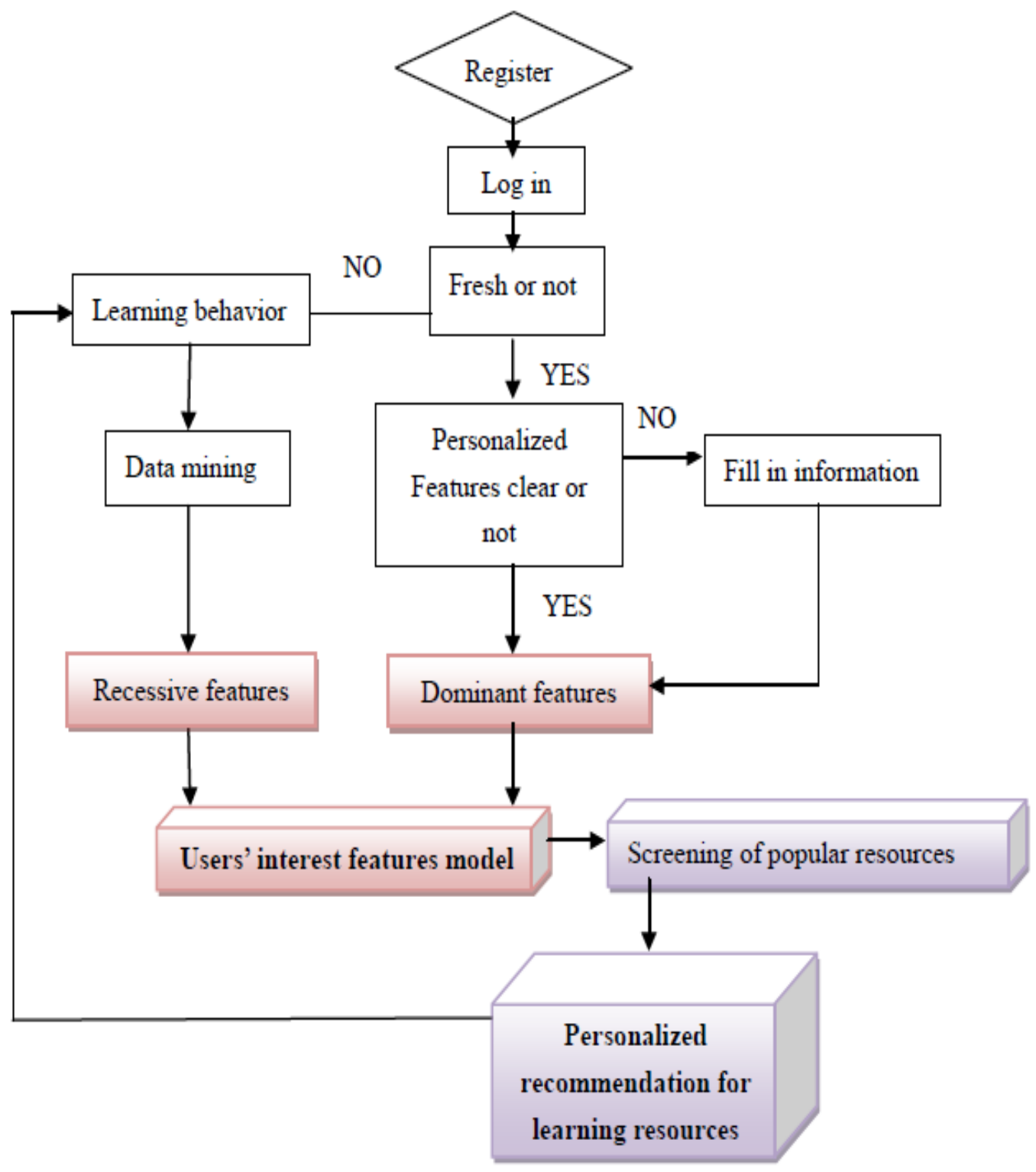

Fig. 3 Personalized recommendation system 
If learner was a fresh user, he needs to choose his learning goal or choose a start point of resource according to own condition. At the same time, the register information will be also used to determine learners' interest feature. Then, the system can make recommendation to learners initially. However, if the learner has registered already, system will make accurate recommendation according to learners' previous learning behavior. Once the learner gets into the learning process, there will be produce behavioral data. System can mining the data exist in weblog and learning log by association rules algorithm to determine learners' interest feature. If the system has learners' interest feature model, recommendation can be made accurately. The recommendation by association rules algorithm can get the correlation exist in resource, so the thematic convergence and integration of resources will be formed for the preparation to make personalized recommendation. The combination of learning resource and personal interest feature is an effective way to build personalized recommendation system.

\section{Conclusion}

With the development of network, E-learning has become the main ways for people to get new knowledge, at the same time, how to improve the efficiency of E-learning has been a serious problem. Rapid selection of useful learning resource for learners can improve the e-learning efficiency undoubtedly. However, if we want to make a selection to learning resources matches with learners' interest, knowing exactly learners' learning style and interest feature is necessary. This paper introduces the data mining technology to dig the behavioral data in order to acquire learners' interest feature. Learners' interest feature is the basis of personalized e-learning system. The popularization and application of personalized e-learning system in the future will improve the quality of network education, and will promote the development of distance education greatly.

\section{References}

[1] Li Ting, Fu Gangshan. Analysis of the situation and trend of data mining at home and abroad[J]. Modern Educational Technology, 2010, 10: 21-25.

[2] Zhou Qing, Mou Chao, Yang Dan. Review of progress for education data mining[J]. Journal of Software, 2015,11:3026-3042.

[3] Peng Wenhui. Analysis and modeling of e-learning behavior[D]. Central China Normal University, 2012.

[4] Huang Ruihang, Qi Liliang. Relational embeddedness: empirical research to online learning behavior[J]. Journal of Distance Education, 2015,06:54-61.

[5][6] Romero, Ventura. Educational data mining: A survey from 1995 to 2005[J]. Expert Systems With Applications, 2007(33),125-146.

[7] Sun Zhongxiang, Peng Xiangjun, Yang Yuping, He Yi. Review of application for data mining in education[J]. Intelligent Computer and Application, 2012,01:78-81.

[8] Jiang Zhihui. The establishment of personalized learning model and strategy optimization in internet environment[J]. Distance education in China, 2013,02:48-51+95. 